In der Rubrik „Literatur kompakt" werden die wichtigsten Originalarbeiten aus der internationalen Fachliteratur referiert.

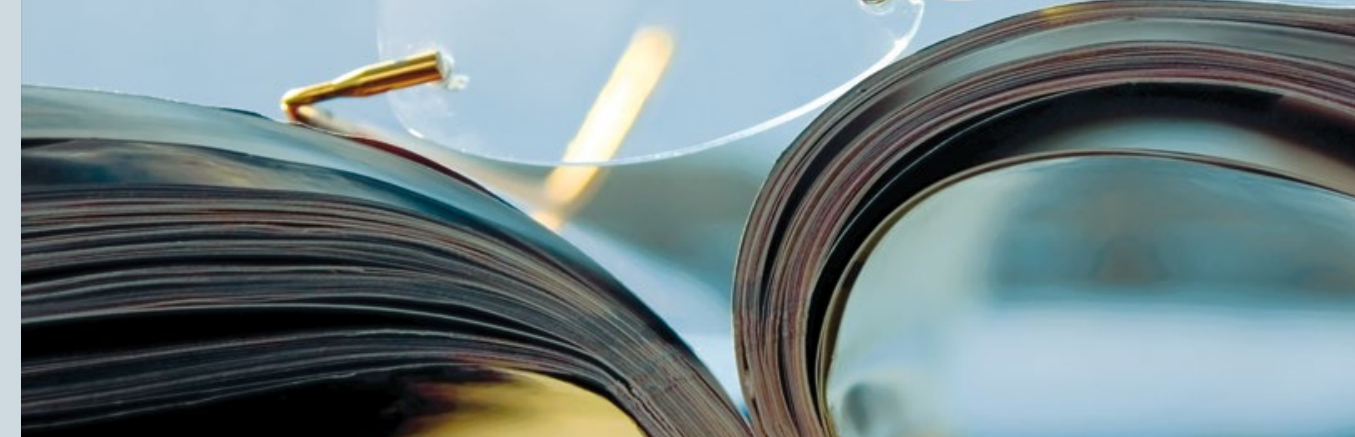

\section{Orale Steroide bei Ischialgie nur wenig wirksam}

\section{Der Einsatz von oralen Steroiden bei akuter Radikulopathie infolge einer lum- balen Diskushernie bessert allenfalls die Funktion, aber nicht den Schmerz.}

aut S2-Leitlinie zur lumbalen Radi- kulopathie kann „die orale Kortikoidgabe in einer Dosis von 50-100 mg Prednisolon pro Tag empirisch insbesondere bei foraminalen Hernien kurzfristig zu einer deutlichen Schmerzreduktion und auch Funktionsverbesserung führen“. Einschränkend wird allerdings darauf hingewiesen, dass „suffiziente Studien, die diesen Eindruck belegen, nicht vor-

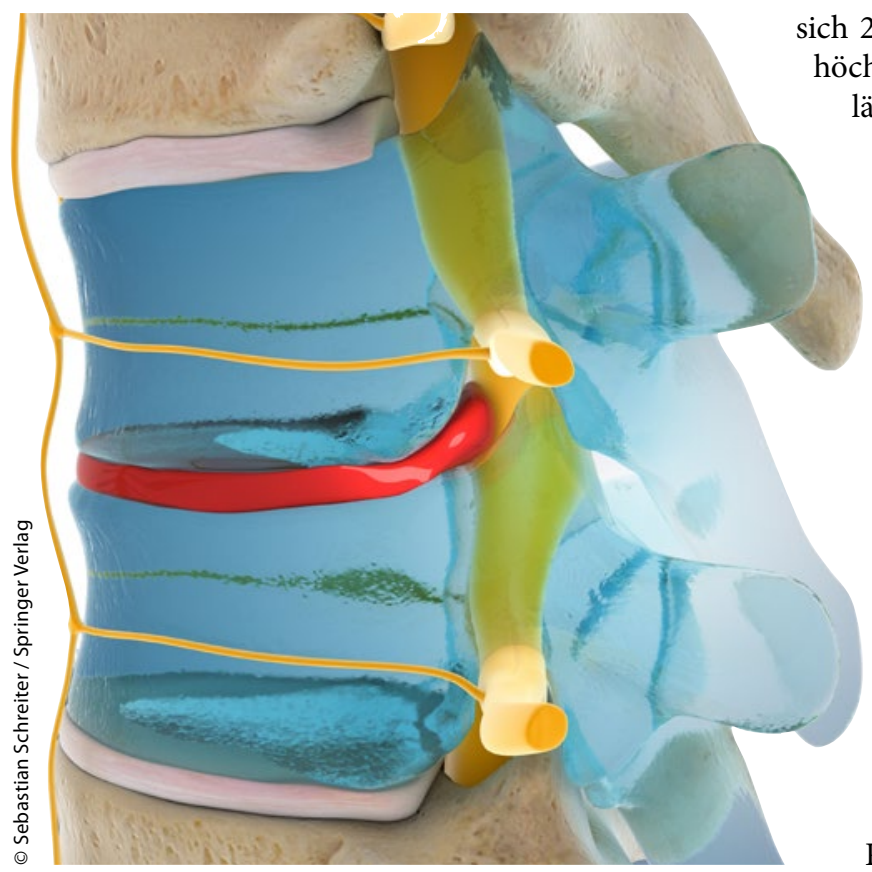

Was tun bei akuten radikulären Schmerzen aufgrund eines Bandscheibenvorfalls? Orales Kortison verordnen? liegen“. Der US-amerikanische Gesundheitsdienstleister Kaiser Permanente Northern California hat eine solche Studie nachgereicht, deren Ergebnisse diesen Eindruck nicht belegen. Die Kurzzeittherapie mit einem oralen Steroid bewirkte eine mäßige Verbesserung der Funktion und keine Verbesserung der Schmerzen, berichten die Studienautoren um Harley Goldberg.

An der randomisierten placebokontrollierten Doppelblindstudie hatten sich 269 Patienten beteiligt, die seit höchstens drei Monaten an radikulären Schmerzen litten, einen per MRT bestätigten Bandscheibenvorfall hatten und im Oswestry Disability Index (ODI) mindestens 30 von schlimmstenfalls $\quad 100$ Punkten aufwiesen. 181 Teilnehmer wurden 15 Tage lang mit absteigenden Dosen Prednison behandelt (jeweils fünf Tage 60, 40 und 20 $\mathrm{mg}), 88$ erhielten ein Placebo.

Der Funktionsstatus gemäß ODI hatte sich nach drei Wochen in beiden Gruppen signifikant verbessert, von 51,2 auf 32,3 Punkte mit Prednison und von 51,1 auf 37,5 Punkte mit Placebo. Nach statistischer Bereinigung ergab dies eine um 6,4 Punkte größere Reduktion in der Steroidgruppe. Die Chance auf einen
Rückgang des ODI-Scores um mindestens 30 Punkte wurde durch das Steroid um $70 \%$ erhöht. Um diese Verbesserung bei einem Patienten zu erreichen, müssten rein rechnerisch 10,6 Patienten behandelt werden. Der Vorteil der Verumgruppe im ODI war nach sechs Wochen verschwunden, überraschenderweise nach einem Jahr aber wieder feststellbar. Die Studienautoren sehen dies als $\mathrm{Zu}$ fallsprodukt, da ein verzögerter Effekt mit der Wirkungsweise des Steroids kaum zu erklären ist. Der Summenscore für die physische Komponente des Lebensqualitätsinstruments Short Form 36 (SF-36) hatte sich nach drei Wochen ebenfalls in der Prednisongruppe stärker gebessert. Nach 52 Wochen war hier kein Unterschied mehr zu erkennen. Keinerlei Einfluss hatte die Steroidtherapie auf die in die Beine ausstrahlenden Schmerzen, die in beiden Gruppen nach drei Wochen und nach einem Jahr gleichermaßen zurückgegangen waren. Außerdem unterzog sich fast jeder zehnte Patient im Lauf des Jahres einer Wirbelsäulen-Op. - unabhängig davon, ob er der Steroidgruppe angehörte oder nicht.

Fazit: Es bleibt die Frage, ob die mit dem Steroid erzielte Veränderung im ODI von 6,4 Punkten auf einer 100-PunkteSkala eine Behandlung begründen kann. Die minimalste Differenz im ODI, die als klinisch relevant angesehen wird, variiert in der Literatur zwischen 5 und 15 Punkten.

Dr. Beate Schumacher

Goldberg $\mathrm{H}$ et al. Oral Steroids for Acute Radiculopathy Due to a Herniated Lumbar Disk. A Randomized Clinical Trial. JAMA2015; 313(19): 19151923; doi:10.1001/jama.2015.4468 